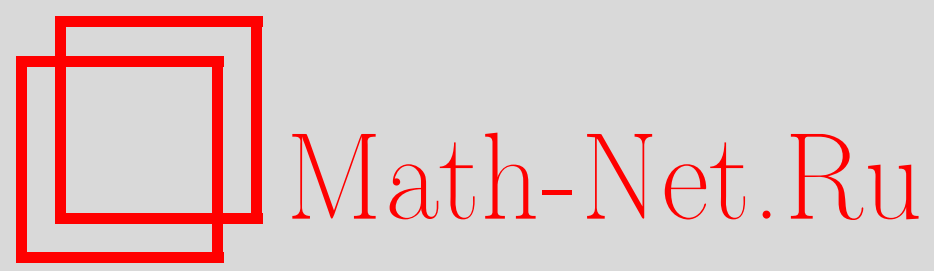

Ю. А. Родичев, К. В. Чарковский, Принципы проектирования корпоративных информационных сетей образовательных учреждений, Вестн. Сам. гос. техн. ун-та. Сер. Физ.-мат. науки, 2003, выпуск 19, 150-155

DOI: https://doi.org/10.14498/vsgtu156

Использование Общероссийского математического портала Math-Net.Ru подразумевает, что вы прочитали и согласны с пользовательским соглашением

http: //www.mathnet.ru/rus/agreement

Параметры загрузки:

IP : 54.209 .52 .79

26 апреля 2023 г., 17:40:08 


\title{
Информатика
}

\author{
УДК 681.3
}

Ю.А. Родичев, К.В. Чарковский

\section{ПРИНЦИПЫ ПРОЕКТИРОВАНИЯ КОРПОРАТИВНЫХ ИНФОРМАЦИОННЫХ СЕТЕЙ ОБРАЗОВАТЕЛЬНЫХ УЧРЕЖДЕНИЙ}

\begin{abstract}
Подчеркивается важность стадии проектирования аппаратной части корпоративных сетей для последующей разработки автоматизированных информационных систем и эффективности внедрения информационных технологий. Предлагаются базовые принципь формирования архитектуpы корпоративных сетей для организаций сферы образования. Дана классификация рабочих станиий сети, состав серверов управления, предложена иерархическая трехуровневая модель построения сети. Описана конкретная реализация указанных принципов на примере корпоративной компьютерной сети Самарского госуниверситета.
\end{abstract}

Непрерывно увеличивающийся объем информации во всех отраслях человеческой деятельности и все возрастающая потребность в ее оперативном и полном получении обусловили активизацию работ в области создания и внедрения компьютерных информационных технологий. Развитие систем телекоммуникаций и использование Internet/Intranet технологий позволили вывести информатизацию на новый качественный уровень. Развитие процессов информатизации и информационной инфраструктуры обеспечивают трансформацию общества из индустриального в информационное. Современные автоматизированные информационные системы (АИС), опираясь на последние достижения в области аппаратно-программных средств и систем телекоммуникаций, дают возможность хранить в базе данных большие объемы информации, поддерживать распределенную обработку данных, обеспечивать доступ к ресурсам системы, как по локальной вычислительной сети (ЛВС), так и через Internet.

Значительный рост вычислительной мощности современных ПЭВМ и увеличение пропускной способности локальных вычислительных сетей обусловили в области учрежденческих информационных технологий тенденцию к переходу от классической централизованной модели обработки данных, основанной на применении дорогостоящих больших ЭВМ, к модели, подразумевающей распределенную обработку централизованно хранящейся информации. Такой подход дает возможность использования в глобальных информационных процессах вычислительных ресурсов ПЭВМ путем частичного переноса алгоритмов обработки данных с центральных ЭВМ (серверов) на компьютеры пользователей (рабочие станции). Основой современных высокоуровневых информационных технологий, применяемых в учрежденческой деятельности, является, как правило, сравнительно небольшой исторически сформировавшийся набор базовых сервисных сетевых подсистем: сетевые файловые системы, служащие для хранения данных общего пользования в виде файлов в иерархически организованных информационных структурах; системы корпоративной электронной почты; системы управления реляционными базами данных, поддерживающие хранение больших объемов однотипной информации в соответствии с четко формализованным и хорошо проработанным набором правил, определяющих методы структурирования и поддержания целостности данных.

Эффективность всей АИС во многом зависит от принципов, заложенных при проектировании аппаратной составляющей - архитектуры корпоративной сети, определяющей набор и функциональное назначение рабочих станций, серверов и средств телекоммуникаций.

Основными признаками, определяющими особенности проектирования структуры сети, являются следующие [1]: размеры охватываемой территории, функциональное назначение и организационная принадлежность, методы распределения информации, топология, виды предоставляемых услуг, однородность структуры. В современных условиях одним из базовых принципов разработки сетей является корпоративность. Это предполагает наличие единой ЛВС организации с выходом в глобальные сети. Методы объединения рабочих станций сети зависят 
от их территориальной удаленности. В пределах здания соединение в основном осуществляется витой парой. Между зданиями сеть строится на основе оптоволоконных линий, либо беспроводных каналов связи, например, технологии “Радио-Ethernet". Важным здесь является принцип, что каждая рабочая станция сети в принципе может иметь доступ ко всем ее ресурсам, включая выход в Internet. Конкретные права доступа определяются средствами системного администрирования и парольной защиты в зависимости от категории пользователей. Кроме того, ЛВС должна предусматривать систему мер безопасности от преднамеренного несанкционированного доступа, а также безопасности данных при возникновении внештатных ситуаций [2].

Функциональное назначение АИС для большинства организаций является стандартным: бухгалтерский учет и финансовое планирование, управление персоналом, подготовка внутренней и внешней отчетности, электронный документооборот. АИС учреждений образования имеют ряд особенностей, связанных с организацией учебного процесса. Эти особенности накладывают ряд требований на архитектуру единой ЛВС и состав серверов управления [3]. По функциональному назначению все рабочие станции сети образовательного учреждения можно разбить на следующие “локальные группы”, образующие первый низший уровень иерархии (“горизонтальные подсистемы”):

1) административно-управленческий аппарат;

2) управление бухгалтерского учета и финансового планирования;

3) управление учебным процессом;

4) автоматизированная библиотечная информационная система (АБИС) с классами доступа к электронным каталогам и полнотекстовой информации на электронных носителях;

5) дисплейные учебные классы общего назначения;

6) классы доступа к мультимедийным электронным учебным курсам (“медиатеки”);

7) классы свободного доступа в Internet;

8) кафедры, лаборатории.

Классы доступа к электронным каталогам организуются при библиотеках и работают под управлением АБИС [4,5]. Учебные классы общего назначения используются для обеспечения учебного процесса по образовательным дисциплинам в области информатики. Классы медиатеки также могут быть расположены на территории библиотеки, но каждая станция должна иметь мультимедийные средства. Все вышеуказанные классы должны иметь выход в Internet и могут в принципе дублировать друг друга. Однако это не всегда целесообразно с финансовой стороны (рабочие станции могут иметь различную конфигурацию, существенно различающуюся по стоимости), а также с точки зрения загрузки трафика межсерверного сегмента сети (каждый тип класса управляется, как правило, своим сервером).

Каждая локальная группа подключается к единой сети с помощью концентраторов. Второй уровень иерархии в структуре ЛВС служит для объединения концентраторов локальных групп в единую систему, выполняя роль “вертикальной подсистемы”. Высший третий уровень иерархии сети образует сегмент серверов общего назначения.

Предлагаемая модель построения ЛВС предусматривает пять классов активных сетевых устройств:

1) выделенные серверы;

2) невыделенные серверы и рабочие станции;

3) маршрутизаторы;

4) концентраторы групп компьютеров.

5) магистральные концентраторы.

К концентраторам групп предъявляются следующие требования: наличие достаточного количества портов для подключения компьютеров группы (звездообразная топология) и 2 порта для подключения к магистральному концентратору. Дополнительный порт может быть использован для разделения трафика в группах, интенсивно использующих сетевые ресурсы, либо как резервный для групп с повышенными требованиями к надежности коммуникаций; возможность организации виртуальных групп с разделенным трафиком, что позволит без изменений в структуре кабельной системы управлять производительностью сети; возможность увеличения количества портов; поддержка протокола SNMP для эффективного мониторинга и управления. К магистральным концентраторам предъявляются требования: пропускная способность внутренней магистрали должна быть не менее удвоенной суммарной пропускной способности всех портов концентратора; возможность мониторинга любого порта и организации виртуальных сетей; поддержка протокола SNMP для эффективного мониторинга и управления; 
возможность наращивания количества портов и установки дополнительных высокопроизводительных модулей.

Принципиальная топология кабельной системы показана на рис. 1. Таким образом, сеть строится в соответствии с принципами централизованного управления с поддержкой рабочих групп. Такая сеть предоставляет возможность организации единого управления сервисами, критичными для работы всех узлов сети (службы идентификации пользователей, безопасности, файловый сервис, электронная почта и т.п.), но не исключает возможность существования ресурсов, принадлежащих только конкретным рабочим группам.

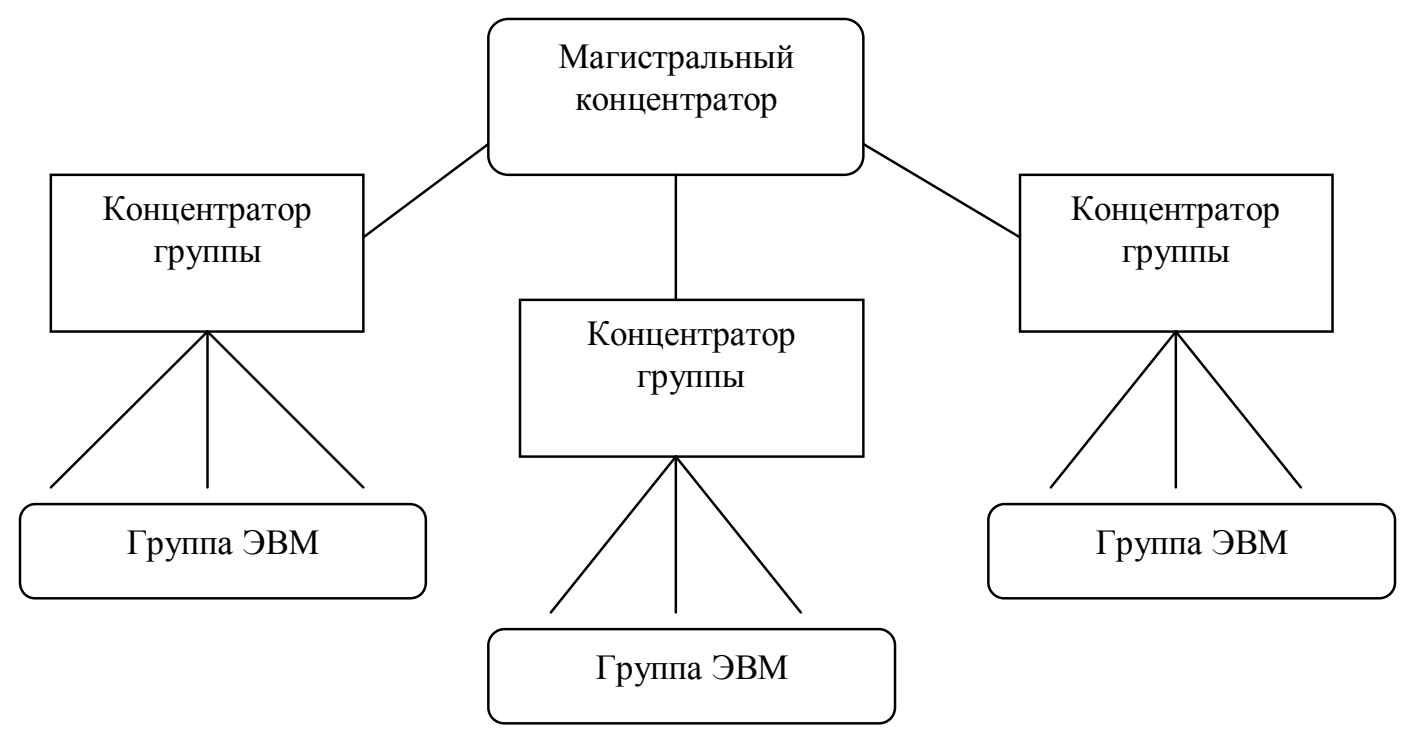

Р и с. 1. Топология кабельной системы

В топологии сети выделяются два класса узлов: выделенные серверы масштаба организации, выделенные и невыделенные серверы и рабочие станции групп. Соответственно узлам выделяется два сегмента ЛВС: сегмент выделенных серверов масштаба организации и сегмент рабочих групп.

Сегмент выделенных серверов масштаба организации объединяет сравнительно небольшое количество мощных серверов и является ядром информационной системы. К нему предъявляются повышенные требования по пропускной способности, стабильности электропитания и условий окружающей среды.

Сегмент рабочих групп объединяет компьютеры, не предъявляющие указанных выше жестких требований. Однако данный сегмент ставит другие проблемы: значительная протяженность коммуникаций; компьютеры, относящиеся к одной рабочей группе, не всегда располагаются в территориальной близости друг от друга; общее количество компьютеров в рабочих группах может быстро расти.

Одним из возможных путей решения указанных проблем является применение сегментируемых наращиваемых концентраторов, позволяющих увеличивать число портов и в широких пределах изменять логическую структуру сети, определяемую рабочими группами, сохраняя неизменным физическое расположение узлов.

В соответствии с вышеизложенными принципами построена единая ЛВС Самарского госуниверситета (рис. 2). В качестве базовых взаимно независимых сетевых служб выделены: многопротокольная универсальная сетевая файловая служба, система управления реляционной базой данных, единая система авторизации доступа. Каждая из названных подсистем поддерживается отдельным сервером или, при повышенных требованиях к производительности и надежности подсистемы, отдельной группой серверов - кластером. Для каждой из указанных служб поддерживается собственная независимая система резервирования данных.

Многопротокольная универсальная сетевая файловая служба (рис.3) поддерживается серверами различной аппаратной архитектуры (IBM PC, Sun SPARC, IBM RS/6000), работающими под управлением UNIX-подобных операционных систем (Solaris, Linux, AIX и т.д.). Выбор ОС данного типа обуславливается высокими показателями в плане универсальности, надежности, управляемости и доступности. При этом взаимодействие серверов между собой, а также с клиентскими компьютерами, имеющими сходные операционные системы, происходит при помощи стандартных средств UNIX (FTP, NFS и т.д.). Обмен с рабочими станциями, работающими под 
управлением отличных от UNIX операционных систем, организуется при помощи виртуальных файловых серверов, поддерживаемых специальными серверными приложениями UNIX, из числа которых можно назвать AIX Connection, Samba и PCNetLink - эмуляторы файл-серверов и контроллеров доменов сети Microsoft Windows (протокол SMBICIFS), а также Mars - эмулятор файл-сервера Novell Netware (протокол NCP).

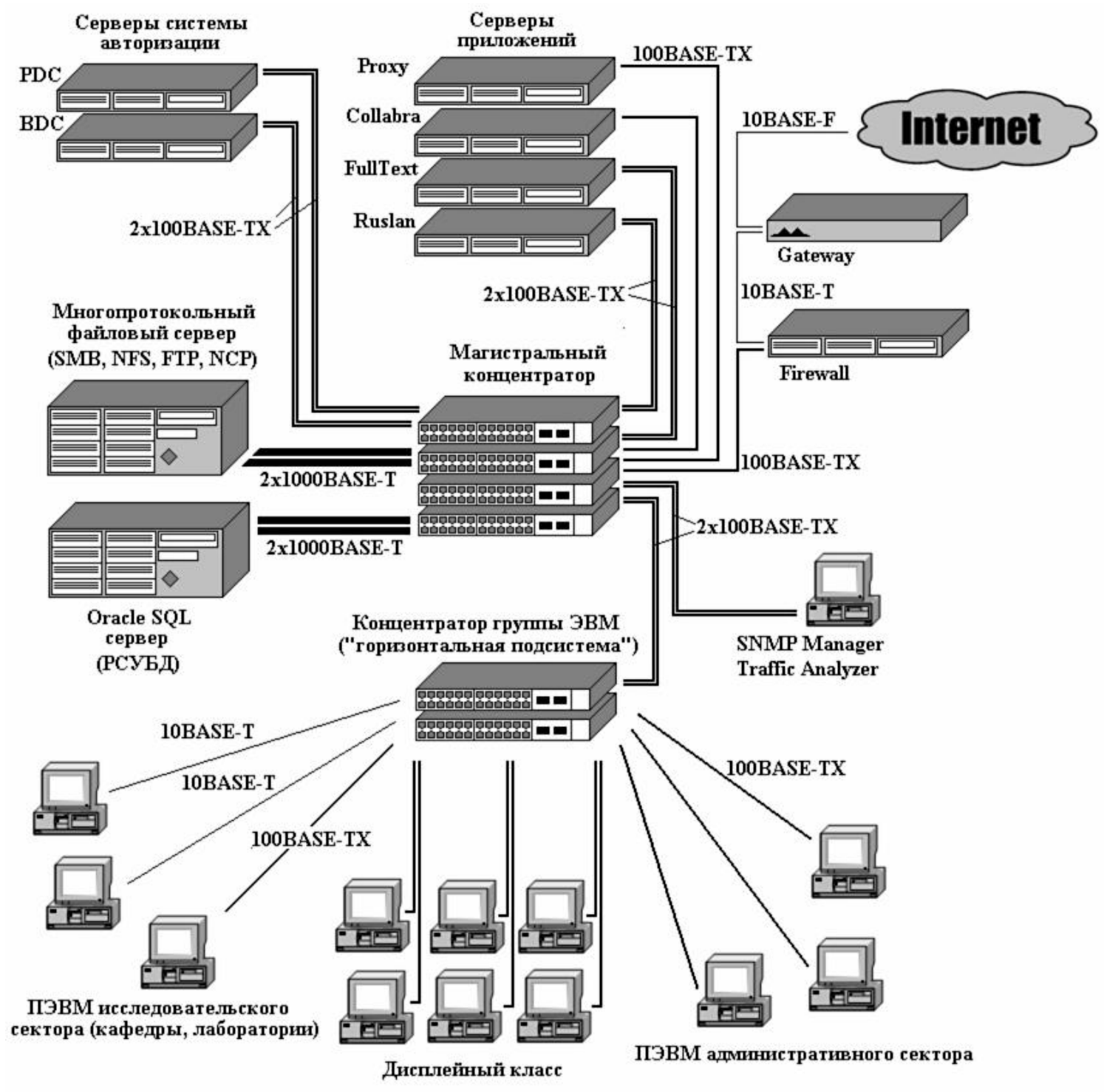

Р и с. 2. Схема единой ЛВС Самарского госуниверситета

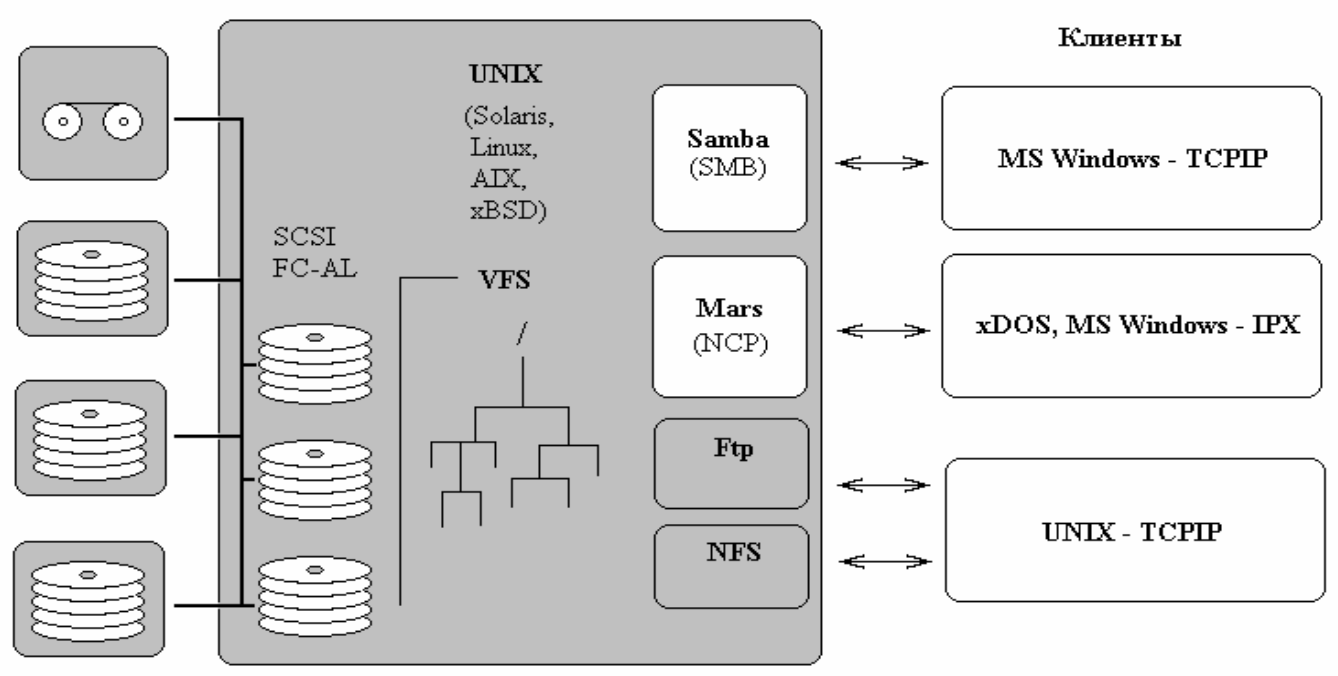

Р и с. 3. Схема универсальной сетевой файловой службы 
Применение виртуальных серверов позволяет построить многоуровневую систему разграничения доступа, более гибкую, чем оригинальные файловые службы OC UNIX или эмулируемых серверов. По сути дела, файл-сервер есть совокупность логических томов, содержащих файловые ресурсы общего пользования. Использование отдельных серверов под управлением оригинальных ОС позволяет разграничивать доступ только при помощи собственных средств ОС файл-сервера. Эмуляция же файл-серверов дает возможность включать дополнительные механизмы, поддерживаемые базовой $\mathrm{OC}$, т.е. той, в среде которой запущены приложения. Кроме того, при использовании оригинальных ОС (Microsoft Windows, Novell Netware) различные серверы должны быть запущены на физически различных компьютерах, следовательно, файловые ресурсы, которые должны быть доступны пользователям разных серверов, должны тиражироваться на все эти серверы, порождая таким образом излишний сетевой трафик, а также создавая проблему своевременного обновления информации. Использование виртуальных файл-серверов, реализованных в виде приложений в среде UNIX-подобной ОС, позволяет легко преодолеть данные ограничения. В этом случае все файловые ресурсы реально размещаются в единой файловой системе, как это и должно быть в UNIX-системах, и один и тот же каталог может быть представлен в виде логических томов в составе различных виртуальных серверов.

На основе многопротокольной универсальной файловой службы в настоящее время функционируют: электронная почта внутреннего назначения, имеющая шлюз в интернет; служба персональных файловых архивов (личные каталоги); сервер персональных web-страниц; общедоступный сетевой архив программного обеспечения и информационных материалов; интерактивные справочные системы различного назначения (СПС “Консультант Плюс" и др.); приложения, поддерживающие деятельность ректората, бухгалтерии, деканатов и других административных подразделений.

Система управления реляционной базой данных является наиболее перспективной в плане развития функциональности. Большинство приложений, создаваемых или приобретаемых организацией для поддержки работы административных подразделений, являются клиентами данной сетевой службы.

Единая система авторизации доступа призвана централизовать хранение учетной информации таким образом, чтобы при запросе пользователем доступа к сетевым ресурсам серверы, поддерживающие запрошенные ресурсы, проверяли информацию об имени и пароле пользователя в единой базе данных. Операционные системы семейства MS Windows отличаются особой "недружелюбностью" к технологиям других производителей, поэтому основой системы авторизации доступа является домен Windows NT, поддерживаемый одним первичным и несколькими резервными контроллерами домена. Операционные системы семейства Unix, напротив, поддерживают самые различные способы идентификации пользователей благодаря технологии PAM. Это позволяет серверам, работающим на платформе Unix, авторизовать пользователей через учетную базу домена MS Windows NT.

Компоненты информационной системы поделены на 4 класса: серверы, поддерживающие функционирование базовых сетевых служб; серверы дополнительных сетевых служб; серверы подразделений, отделов, невыделенные серверы и рабочие станции с повышенными требованиями к пропускной способности сети; обычные рабочие станции.

Классы абонентов перечислены в порядке понижения требований по качеству обслуживания. Для абонентов 1-го класса ЛВС должна обеспечивать наивысшую доступность в смысле скорости передачи данных и надежности подключения, абоненты 2-го класса более многочисленны, но предъявляют несколько более низкие требования, в чем с очевидностью прослеживается многоуровневая иерархическая природа информационных систем с централизованным хранением и распределенной обработкой. Физическая топология ЛВС, которая обязана учитывать приведенную классификацию компонентов ИС, в данном случае имеет иерархическое строение, а именно: “двухуровневая звезда". При этом на первом уровне “звезды” обеспечивается подключение сравнительно немногочисленных компонентов 1-го и 2-го классов и “звезд” 2-го уровня, которые обеспечивают подключение компонентов 3-го и 4-го классов. Технологии ЛВС наиболее распространенного семейства Ethernet позволяют предложить решение, удовлетворительное для каждого класса абонентов, а именно: 1-й класс - объединенные с целью повышения производительности и надежности полнодуплексные коммутируемые соединения GigabitEthernet и FastEthernet с автоматическим перераспределением нагрузки; 2-й класс - выделенные полнодуплексные коммутируемые соединения GigabitEthernet и FastEthernet; 3-й класс - полнодуплексные коммутируемые соединения FastEthernet и Ethernet; 4-й класс - полудуплексные соединения FastEthernet и Ethernet. 
Выполнение указанных требований в настоящее время обеспечивается управляемыми наращиваемыми коммутаторами семейства 3Com SuperStack 3.

\section{БИБЛИОГРАФИЧЕСКИЙ СПИСОК.}

1. Морозов В.К., Долганов А.В. Основы теории информационных сетей. М.: Высш. шк. 1987. $257 \mathrm{c}$.

2. Морозов А.В., Родичев Ю.А. Сохранность ресурсов автоматизированных информационных финансовоэкономических и бухгалтерских систем // Измерение, контроль, информатизация ИКИ-2000: Тез. докл. Междунар. науч.-техн. конф. Барнаул, 2000. С. 148-151.

3. Родичев Ю.А., Чарковский К.В. Практический опыт создания и применения сетевых информационных систем для автоматизации управленческой деятельности вуза // Телематика-97: Тез. докл. Всерос. науч. - методич. конф. СПб, 1997. С. 101-102.

4. Шрайберг Я.Л. Основные положения и принципы разработки автоматизированных библиотечноинформационных систем и сетей. М.: Физматлит, 2001. 340 с.

5. Воройский Ф.С. Основы проектирования автоматизированных библиотечно-информационных систем. М.: Физматлит, 2002. $242 \mathrm{c}$.

Поступила 20.02.2003 г. 